Neoclassical Transport Coefficients for General Axisymmetric Equilibria in the Banana Regime

C. Angioni, O. Sauter

A shorter version will be submitted for publication in PHYSICS OF PLASMAS 


\title{
Neoclassical transport coefficients for general axisymmetric equilibria in the banana regime
}

\author{
C. Angioni*, O. Sauter \\ Centre de Recherches en Physique des Plasmas \\ Association EURATOM - Confédération Suisse, \\ Ecole Polytechnique Fédérale de Lausanne, 1015 Lausanne, Switzerland
}

\begin{abstract}
Using the standard approach of neoclassical theory, a set of relatively simple kinetic equations has been obtained, suited for an implementation in a numerical code to compute a related set of distribution functions. The transport coefficients are then expressed by simple integrals of these functions and they can be easily computed numerically. The code CQL3D, which uses the full collision operator and considers the realistic axisymmetric configuration of the magnetic surfaces, has been modified to solve the bounce-averaged version of these equations. The coefficients have then been computed for a wide variety of equilibrium parameters, highlighting interesting features of the influence of geometry at small aspect ratio. Differences with the most recent formulas for the ion neoclassical heat conductivity are pointed out. A set of formulas, which fit the code results, is proposed to easily evaluate all the neoclassical transport coefficients in the banana regime, at all aspect ratios, in general axisymmetric equilibria. This work extends to all the other transport coefficients, at least in the banana regime, the work of Sauter et al. [O. Sauter, C. Angioni and Y. R. LinLiu, Phys. Plasmas 62834 (1999)] which evaluates the neoclassical conductivity and all the bootstrap current coefficients. Tentative formulas for arbitrary collisionality regime are suggested, obtained adapting the results of Hinton and Hazeltine [F. L. Hinton and R. D. Hazeltine, Rev. Mod. Phys. 48, 239 (1976)].
\end{abstract}

\footnotetext{
*Corresponding author, email address: Clemente.Angioni@epfl.ch
} 


\section{INTRODUCTION}

Recent improvements in neoclassical transport theory are almost completely dedicated to parallel transport. In particular, in Ref. 1, we have computed the neoclassical conductivity and all the bootstrap current coefficients, taking into account the full collision operator and including the advection parallel to the magnetic field, considering the realistic axisymmetric magnetic configuration of the flux surface. We have given relatively simple formulas valid for general axisymmetric equilibria and arbitrary collisionality regimes. For the other transport coefficients, improvements have been done only for the ion thermal conductivity in the banana regime ${ }^{3-5}$ and for various collision frequencies ${ }^{6}$. All these latter results, however, use an approximated version of the collision operator, usually following the expansion of Hirshman and Sigmar ${ }^{7}$. For all the other transport coefficients the only formulas available at small aspect ratio are those in Ref. 9, valid in the banana regime, which use the analytical values of the transport coefficients at $\epsilon=1$ and the values at large aspect ratio of Ref. 10 to obtain a set of formulas with a linear interpolation between these two limits, which should be valid also at small aspect ratio. In the more recent investigations on the ion thermal conductivity ${ }^{4-5}$, the intermediate aspect ratio corrections show a difference with the results of Ref. 9 of almost a factor of two. In this sense a complete investigation of the small aspect ratio corrections for all the neoclassical transport coefficients, taking into account the full collision operator, is necessary. It is well known that the neoclassical theory can not explain the perpendicular transport in tokamaks, however a precise computation is useful in order to allow a correct evaluation of the anomalous contribution by means of the comparison with the experimental data. This is becoming even more important with the recent improved confinement modes of operation, with internal transport barriers and relatively small anomalous transport.

In Sec. II we describe the approach to obtain the linear drift-kinetic equations suitable for implementation in a Fokker-Planck code and the expressions to compute the transport coefficients as simple integrals of the distribution functions. The related bounce-averaged 
equations in the banana regime are then obtained, and the Lorentz model is investigated analytically. In Sec. III we show the numerical results for the banana regime, computed with the Fokker-Planck code $\mathrm{CQL} \mathrm{D}^{8}$, which solves the linearized drift kinetic bounceaveraged equation with the full collision operator and considering the realistic axisymmetric configuration of the magnetic surfaces. Some benchmarks are considered to validate the results, and the comparison with some previous numerical and analytical results is shown. In Sec. IV we give a set of formulas which fit our numerical results and allow to easily evaluate all the neoclassical transport coefficients in general axisymmetric equilibria for arbitrary aspect ratio and ion charge in the banana regime. Tentative formulas for arbitrary collisionality regime are then suggested in the last subsection.

\section{KINETIC THEORY}

\section{A. Transport Coefficients}

Our approach follows the standard neoclassical theory, in particular the one of Ref. 2. Definitions of thermodynamic forces and fluxes are only slightly different. We begin considering the Linearized Drift Kinetic Fokker-Planck equations, Ref. 2, Eqs. (5.21-24):

$$
\begin{aligned}
& v_{\|} \hat{\mathbf{b}} \cdot \nabla f_{e 1}-C_{e}^{l}\left(f_{e 1}\right)=-\left(v_{D} \cdot \nabla \psi\right)_{e} \frac{\partial f_{e 0}}{\partial \psi}-\frac{q_{e} E_{\|}}{m_{e}} \frac{\partial f_{e 0}}{\partial v_{\|}} \\
& v_{\|} \hat{\mathbf{b}} \cdot \nabla f_{i 1}-C_{i i}^{l}\left(f_{i 1}\right)=-\left(v_{D} \cdot \nabla \psi\right)_{i} \frac{\partial f_{i 0}}{\partial \psi}
\end{aligned}
$$

with

$$
\begin{aligned}
& C_{e}^{l}=C_{e e}^{l}+C_{e i}^{l} \quad \text { linearized collision operator, } \\
& \left(v_{D} \cdot \nabla \psi\right)_{\sigma}=I(\psi) v_{\|} \hat{\mathbf{b}} \cdot \nabla\left(\frac{v_{\|}}{\Omega_{\sigma}}\right), \\
& \frac{\partial f_{\sigma 0}}{\partial \psi}=f_{\sigma 0}\left[\frac{\partial \ln n_{\sigma 0}}{\partial \psi}+\frac{q_{\sigma}}{T_{\sigma}} \frac{\partial\langle\Phi\rangle}{\partial \psi}+\left(\frac{v^{2}}{v_{T_{\sigma}}^{2}}-\frac{3}{2}\right) \frac{\partial \ln T_{\sigma 0}}{\partial \psi}\right],
\end{aligned}
$$

and where $v_{T \sigma}^{2}=2 T_{\sigma}^{2} / m_{\sigma}$ and $\Omega_{\sigma}=q_{\sigma} B / m_{\sigma}$ are the thermal velocity and the cyclotron frequency of species $\sigma, I(\psi)=R B_{\phi}, \hat{\mathbf{b}}=\mathbf{B} / B$, and $\langle\Phi\rangle$ is the flux surface averaged elec- 
trostatic potential. Similar to the derivation presented in Ref. 2, we perform the following transformation of the distribution functions:

$$
\begin{aligned}
& f_{e 1}=\frac{2 v_{\|} u_{i \|}}{v_{T e}^{2}} f_{e 0}+\frac{B\left\langle E_{\|} B\right\rangle}{\left\langle B^{2}\right\rangle} v_{\|} f_{s e}+\frac{q_{e}}{T_{e}} f_{e 0} \int_{0}^{l_{p}} \frac{d l_{p}}{B_{p}}\left(B E_{\|}-\frac{B^{2}\left\langle E_{\|} B\right\rangle}{\left\langle B^{2}\right\rangle}\right)+H_{e}, \\
& f_{i 1}=-\frac{I(\psi) v_{\|}}{\Omega_{i}}\left(\frac{\partial \ln p_{i}}{\partial \psi}+\frac{q_{i}}{T_{i}} \frac{\partial\langle\Phi\rangle}{\partial \psi}\right) f_{i 0}+\frac{q_{i}}{T_{i}} f_{i 0} \int_{0}^{l_{p}} \frac{d l_{p}}{B_{p}}\left(B E_{*}-\frac{B^{2}\left\langle E_{*} B\right\rangle}{\left\langle B^{2}\right\rangle}\right)+H_{i},
\end{aligned}
$$

where

$$
u_{i \|}=\frac{K_{i}(\psi) B}{n_{i}}-\frac{I(\psi)}{B}\left(\frac{T i}{q_{i}} \frac{\partial \ln p_{i}}{\partial \psi}\right)+\frac{\partial\langle\Phi\rangle}{\partial \psi},
$$

\langle\rangle denotes the flux surface average, $f_{s e}$ is the Spitzer function and $K_{i}(\psi)$ is a function of the magnetic poloidal flux $\psi$ which will be determined later; $E_{*} \doteq E_{\|}+F_{i \|}\left(q_{i} n_{i}\right)^{-1}$ is the so-called "effective electric field" and $F_{i||}$ is the friction force between ions and electrons. For the new distribution functions $H_{e}$ and $H_{i}$, the LDK equations can be written in the following "canonical" form:

$$
\begin{aligned}
& v_{\|} \hat{\mathbf{b}} \cdot \nabla H_{e}-C_{e 0}^{l}\left(H_{e}\right)=-\sum_{n} v_{\|} \hat{\mathbf{b}} \cdot \nabla\left(\gamma_{e n}\right) A_{e n} f_{e 0}, \\
& v_{\|} \hat{\mathbf{b}} \cdot \nabla H_{i}-C_{i i}^{l}\left(H_{i}\right)=-\sum_{n} \beta_{i n} A_{i n} f_{i 0},
\end{aligned}
$$

where we have introduced the flux surface averaged thermodynamic forces, as follows:

$$
\begin{array}{rlrl}
A_{e 1} & =\frac{1}{p_{e}} \frac{\partial p_{e}}{\partial \psi}+\frac{1}{p_{e}} \frac{\partial p_{i}}{\partial \psi}, & & \\
A_{e 2} & =\frac{1}{T_{e}} \frac{\partial T_{e}}{\partial \psi}, & A_{i 1} & =-\frac{\left\langle E_{*} B\right\rangle}{\left\langle B^{2}\right\rangle} \\
A_{e 3} & =\frac{\left\langle E_{\|} B\right\rangle}{\left\langle B^{2}\right\rangle}, & A_{i 2} & =\frac{1}{T_{i}} \frac{\partial T_{i}}{\partial \psi} \\
A_{e 4} & =\frac{q_{e} K_{i}(\psi)\left\langle B^{2}\right\rangle}{n_{i} I(\psi) T_{e}} . &
\end{array}
$$

We have also introduced the following functions:

$$
\begin{aligned}
\gamma_{e 1}=\frac{I(\psi) v_{\|}}{\Omega_{e}}, & \gamma_{e 2}=\gamma_{e 1}\left(\frac{v^{2}}{v_{T e}^{2}}-\frac{5}{2}\right) \\
\gamma_{e 3}=\frac{v_{\|} f_{s e}}{f_{e 0}} B, & \gamma_{e 4}=\gamma_{e 1} \frac{B^{2}}{\left\langle B^{2}\right\rangle} \\
\beta_{i 1}=\frac{q_{i} v_{\|}}{T_{i}} B, & \beta_{i 2}=v_{\|} \hat{\mathbf{b}} \cdot \nabla\left(\gamma_{i 2}\right), \\
\gamma_{i 2}= & \frac{I(\psi) v_{\|}}{\Omega_{i}}\left(\frac{v^{2}}{v_{T i}^{2}}-\frac{5}{2}\right) .
\end{aligned}
$$


The conjugated neoclassical thermodynamic fluxes are identified in the expression of the linearized entropy production ${ }^{2}$, and can be defined as follows:

$$
\begin{array}{ll}
B_{e n}=\left\langle\int d \mathbf{v}\left(v_{\|} \hat{\mathbf{b}} \cdot \nabla \gamma_{e n}\right) H_{e}\right\rangle, & n=1,2,3,4, \\
B_{i n}=\left\langle\int d \mathbf{v} \beta_{i n} H_{i}\right\rangle, & n=1,2,
\end{array}
$$

which gives, with $\rho$ the generic radial coordinate:

$$
\begin{aligned}
B_{e 1}=\Gamma_{e} \frac{d \psi}{d \rho}, & B_{e 2}=\frac{Q_{e}}{T_{e}} \frac{d \psi}{d \rho} \\
B_{e 3}=\frac{\left\langle j_{\|} B\right\rangle}{T_{e}}-\frac{\left\langle j_{\| S} B\right\rangle}{T_{e}}, & B_{e 4}=-\frac{\left\langle E_{*} B\right\rangle}{\left\langle B^{2}\right\rangle} I(\psi) n_{e} \\
B_{i 1}=\frac{\left\langle j_{\| R i} B\right\rangle}{T_{i}}, & B_{i 2}=\frac{Q_{i}}{T_{i}} \frac{d \psi}{d \rho}
\end{aligned}
$$

where $\Gamma_{\sigma}$ and $Q_{\sigma}$ are the particle and heat fluxes of species $\sigma, j_{\|}$is the total parallel electric current, $j_{\| S}$ is the so-called Spitzer current and $j_{\| R i}$ is the ion contribution to the so-called "return current". The flux surface average $\left\langle j_{\| R i} B\right\rangle$ is related to the function $K_{i}(\psi)$ by the following equation:

$$
\left\langle j_{\| R i} B\right\rangle=q_{i} K_{i}(\psi)\left\langle B^{2}\right\rangle
$$

Note that ion and electron forces and fluxes are mutually dependent:

$$
B_{i 1}=-I(\psi) n_{e} \frac{T_{e}}{T_{i}} A_{e 4}, \quad B_{e 4}=I(\psi) n_{e} A_{i 1}
$$

The transport coefficients linearly relate forces with fluxes, $B_{\sigma m}=\sum_{n} \mathcal{L}_{m n}^{\sigma} A_{\sigma n}$. To easily identify the role of each transport coefficient, we show the complete relation between electron and ion forces and fluxes in the following form:

$$
\left(\begin{array}{c}
\Gamma_{e} \frac{d \psi}{d \rho} \\
\frac{Q_{e}}{T_{e}} \frac{d \psi}{d \rho} \\
\left\langle\frac{j_{\|} B}{T_{e}}\right\rangle-\left\langle\frac{j_{\| S} B}{T_{e}}\right\rangle \\
-\frac{I(\psi)\left\langle E_{*} B\right\rangle n_{e}}{\left\langle B^{2}\right\rangle}
\end{array}\right)=\left[\begin{array}{cccc}
\mathcal{L}_{11}^{e} & \mathcal{L}_{12}^{e} & \mathcal{L}_{13}^{e} & \mathcal{L}_{14}^{e} \\
\mathcal{L}_{21}^{e} & \mathcal{L}_{22}^{e} & \mathcal{L}_{23}^{e} & \mathcal{L}_{24}^{e} \\
\mathcal{L}_{31}^{e} & \mathcal{L}_{32}^{e} & \mathcal{L}_{33}^{e} & \mathcal{L}_{34}^{e} \\
\mathcal{L}_{41}^{e} & \mathcal{L}_{42}^{e} & \mathcal{L}_{43}^{e} & \mathcal{L}_{44}^{e}
\end{array}\right]\left(\begin{array}{c}
\frac{1}{p_{e}} \frac{\partial p_{e}}{\partial \psi}+\frac{1}{p_{e}} \frac{\partial p_{i}}{\partial \psi} \\
\frac{1}{T_{e}} \frac{\partial T_{e}}{\partial \psi} \\
\frac{\left\langle E_{\|} B\right\rangle}{\left\langle B^{2}\right\rangle} \\
\frac{q_{e}}{n_{i} T_{e}} \frac{K_{i}(\psi)}{I(\psi)}\left\langle B^{2}\right\rangle
\end{array}\right)
$$




$$
\left(\begin{array}{c}
\frac{\left\langle j_{\| R i} B\right\rangle}{T_{i}} \\
\frac{Q_{i}}{T_{i}} \frac{d \psi}{d \rho}
\end{array}\right)=\left[\begin{array}{ll}
\mathcal{L}_{11}^{i} & \mathcal{L}_{12}^{i} \\
\mathcal{L}_{21}^{i} & \mathcal{L}_{22}^{i}
\end{array}\right]\left(\begin{array}{c}
-\frac{\left\langle E_{*} B\right\rangle}{\left\langle B^{2}\right\rangle} \\
\frac{1}{T_{i}} \frac{\partial T_{i}}{\partial \psi}
\end{array}\right)
$$

We now perform the second transformation:

$$
\begin{aligned}
G_{e} & =H_{e}+\sum_{n} \gamma_{e n} A_{e n} f_{e 0}, \\
G_{i} & =H_{i}+\gamma_{i 2} A_{i 2} f_{i 0} .
\end{aligned}
$$

The LDK equations for the new distribution functions are:

$$
\begin{aligned}
& v_{\|} \hat{\mathbf{b}} \cdot \nabla G_{e}-C_{e 0}^{l}\left(G_{e}\right)=-\sum_{n} C_{e 0}^{l}\left(\gamma_{e n} f_{e 0}\right) A_{e n}, \\
& v_{\|} \hat{\mathbf{b}} \cdot \nabla G_{i}-C_{e 0}^{l}\left(G_{i}\right)=-\beta_{i 1} f_{i 0} A_{i 1}-C_{i i}^{l}\left(\gamma_{i 2} f_{i 0}\right) A_{i 2} .
\end{aligned}
$$

Note that now all the coefficients of the thermodynamic forces in the source terms can be evaluated analytically ${ }^{11}$ :

$$
\begin{aligned}
-C_{e 0}^{l}\left(\gamma_{e 1} f_{e 0}\right) & =Z_{i} \nu_{e 0}(v) \gamma_{e 1} f_{e 0}, \\
-C_{e 0}^{l}\left(\gamma_{e 2} f_{e 0}\right) & =Z_{i} \nu_{e 0}(v) \gamma_{e 2} f_{e 0}-\nu_{e 0}(v) h\left(\frac{v}{v_{T e}}\right) \gamma_{e 1} f_{e 0}, \\
-C_{e 0}^{l}\left(\gamma_{e 3} f_{e 0}\right) & =\frac{q_{e} v_{\|} B}{T_{e}} f_{e 0}, \\
-C_{e 0}^{l}\left(\gamma_{e 4} f_{e 0}\right) & =Z_{i} \nu_{e 0}(v) \gamma_{e 4} f_{e 0}, \\
-C_{i i}^{l}\left(\gamma_{i 2} f_{e 0}\right) & =-\nu_{i 0}(v) h\left(\frac{v}{v_{T i}}\right) \frac{I(\psi) v_{\|}}{\Omega_{i}} f_{i 0},
\end{aligned}
$$

with

$$
\begin{array}{cr}
\nu_{e 0}(v)=\frac{3 \sqrt{\pi} v_{T e}^{3}}{4 Z_{i} \tau_{e} v^{3}}, & \nu_{i 0}(v)=\frac{3 \sqrt{\pi} v_{T i}^{3}}{2 \sqrt{2} \tau_{i} v^{3}}, \\
h(x) & =\left(10-4 x^{2}\right) \operatorname{erf}(x)-10 x \operatorname{erf}^{\prime}(x) .
\end{array}
$$

As the equations are linear and the thermodynamic forces can be treated as independent, we can write the unknown function $G_{e}$ for the electrons as a linear combination in the following form: $G_{e}=\sum_{n} g_{e n} A_{e n}$. For the set of functions $g_{e n}$, Eq. (7) can be decoupled, leading to the following set of equations:

$$
v_{\|} \hat{\mathbf{b}} \cdot \nabla g_{e n}-C_{e 0}^{l}\left(g_{e n}\right)=-C_{e 0}^{l}\left(\gamma_{e n} f_{e 0}\right), \quad n=1,2,3,4 .
$$


In the same way, introducing $G_{i}=\sum_{n} g_{i n} A_{i n}$ in Eq. (8) for the ions, we obtain:

$$
\begin{aligned}
& v_{\|} \hat{\mathbf{b}} \cdot \nabla g_{i 1}-C_{i i}^{l}\left(g_{i 1}\right)=-\beta_{i 1} f_{i 0} \\
& v_{\|} \hat{\mathbf{b}} \cdot \nabla g_{i 2}-C_{i i}^{l}\left(g_{i 2}\right)=-C_{i i}^{l}\left(\gamma_{i 2} f_{i 0}\right)
\end{aligned}
$$

We look now at the definitions of the thermodynamic fluxes, Eqs.(3) and (4); a simple calculation shows that for the electrons we can write:

$$
B_{e n}=\sum_{m}\left[\left\langle\int d \mathbf{v} \gamma_{e m} C_{e 0}^{l}\left(\gamma_{e n} f_{e 0}\right)\right\rangle-\left\langle\int d \mathbf{v} \frac{g_{e m}}{f_{e 0}} C_{e 0}^{l}\left(\gamma_{e n} f_{e 0}\right)\right\rangle\right] A_{e m}
$$

Analogously the ion fluxes read:

$$
\begin{aligned}
B_{i 1} & =\sum_{n}\left\langle\int d \mathbf{v} \beta_{i 1} g_{i n}\right\rangle A_{i n} \\
B_{i 2} & =\left\langle\int d \mathbf{v} \gamma_{i 2} C_{e 0}^{l}\left(\gamma_{i 2} f_{i 0}\right)\right\rangle A_{i 2}-\sum_{m}\left\langle\int d \mathbf{v} \frac{g_{i m}}{f_{i 0}} C_{i i}^{l}\left(\gamma_{i 2} f_{i 0}\right)\right\rangle A_{i m} .
\end{aligned}
$$

At this point we can identify the transport coefficients:

$$
\begin{aligned}
\mathcal{L}_{m n}^{e} & =\left\langle\int d \mathbf{v} \gamma_{e m} C_{e 0}^{l}\left(\gamma_{e n} f_{e 0}\right)\right\rangle-\left\langle\int d \mathbf{v} \frac{g_{e m}}{f_{e 0}} C_{e 0}^{l}\left(\gamma_{e n} f_{e 0}\right)\right\rangle, \quad n, m=1,2,3,4 \\
\mathcal{L}_{1 n}^{i} & =\left\langle\int d \mathbf{v} g_{i n} \beta_{i 1}\right\rangle, \quad n=1,2 \\
\mathcal{L}_{21}^{i} & =-\left\langle\int d \mathbf{v} \frac{g_{i 1}}{f_{i 0}} C_{i i}^{l}\left(\gamma_{i 2} f_{i 0}\right)\right\rangle \\
\mathcal{L}_{22}^{i} & =\left\langle\int d \mathbf{v} \gamma_{i 2} C_{i i}^{l}\left(\gamma_{i 2} f_{i 0}\right)\right\rangle-\left\langle\int d \mathbf{v} \frac{g_{i 2}}{f_{i 0}} C_{i i}^{l}\left(\gamma_{i 2} f_{i 0}\right)\right\rangle
\end{aligned}
$$

In this way we have obtained a simple set of expressions to compute all the neoclassical transport coefficients, once we have solved the drift-kinetic equations, Eqs. (10) and (11), to obtain the distribution functions $g_{e n}$ and $g_{i n}$. We see that every expression is composed of the sum of two terms: the first one is an integral that can be computed analytically and that, for some coefficients, is identically zero; the second one has an integrand in which the only term to be computed numerically is the distribution function $g_{e n}$ or $g_{i n}$. Note that in the banana regime the first term, computed analytically, gives directly the value of the transport coefficient at $\epsilon=1$, when all the particles are trapped; the second term gives the reduction of transport due to the presence of passing particles. In Appendix A we show that Eqs. (12) and Eqs. (13) satisfy the Onsager relations of symmetry as expected. 


\section{B. Banana Regime: Bounce-Averaged Equations}

When the collision frequency $\nu_{e i}$ is much smaller than the bounce frequency $\nu_{b}$, the distribution functions $g_{e n}$ can be expanded as follows:

$$
g_{e n}=g_{e n}^{0}+\left(\frac{\nu_{e i}}{\nu_{b}}\right) g_{e n}^{1}+O\left[\left(\frac{\nu_{e i}}{\nu_{b}}\right)^{2}\right]
$$

and analogously for the ion distribution functions. A somewhat standard derivation ${ }^{2,10}$ shows that the functions $g_{\sigma n}^{0}$ are independent of the poloidal angle $\theta_{p}$, and that they are zero in the trapped particle region of velocity space.

In the passing particle region, the functions $g_{\sigma n}^{0}$ satisfy the following bounce-averaged equations:

$$
\begin{array}{ll}
\int_{-\pi}^{\pi} d \theta_{p} \frac{B}{\left|v_{\|}\right|} C_{e 0}^{l}\left(g_{e n}^{0}\right)=S_{e n}, & n=1,2,3,4, \\
\int_{-\pi}^{\pi} d \theta_{p} \frac{B}{\left|v_{\|}\right|} C_{i i}^{l}\left(g_{i n}^{0}\right)=S_{i n}, & n=1,2,
\end{array}
$$

with:

$$
\begin{aligned}
& S_{e n} \doteq 2 \pi \sigma\left\langle B \mathcal{C}_{e n}^{\gamma}(v, B)\right\rangle f_{e 0}, \quad n=1,2,3,4, \\
& S_{i 1} \doteq 2 \pi \sigma \frac{q_{i}\left\langle B^{2}\right\rangle}{T_{i}} f_{i 0}, \quad S_{i 2} \doteq 2 \pi \sigma\left\langle B \mathcal{C}_{i 2}^{\gamma}(v, B)\right\rangle f_{i 0},
\end{aligned}
$$

where $\sigma=v_{\|} /\left|v_{\|}\right|$and where we have introduced the set of functions $\mathcal{C}_{\sigma n}^{\gamma}(v, B)$, defined as follows:

$$
\mathcal{C}_{\sigma n}^{\gamma}(v, B) \doteq C_{\sigma}^{l}\left(\gamma_{\sigma n} f_{\sigma 0}\right) /\left(v_{\|} f_{\sigma 0}\right)
$$

The analytical expressions of these functions can easily be obtained from Eqs. (9). Note that $\left\langle B \mathcal{C}_{e 4}^{\gamma}(v, B)\right\rangle=\left\langle B \mathcal{C}_{e 1}^{\gamma}(v, B)\right\rangle$, so that the functions $g_{e 1}^{0}$ and $g_{e 4}^{0}$ solve the same equation in the banana regime; in particular it follows that $\mathcal{L}_{44}=\mathcal{L}_{14}$ : this is a consequence of our choice of thermodynamic forces and fluxes. We see that at $\epsilon=1$, when all the particles are trapped, the distribution functions $g_{e n}^{0}$ are zero everywhere, and the first terms in the expressions for the transport coefficients, Eqs. (12) and (13), give directly the entire coefficient. The code CQL3D has been modified to solve Eqs. (14) in general axisymmetric equilibria and with the full collision operator. 


\section{Lorentz Model}

For the Lorentz gas model, $Z_{i} \gg 1$, the set of Eqs. (14a) is solved analytically ${ }^{2,12}$. In fact, as collisions between electrons can be neglected, the collision operator can be approximated by the pitch-angle scattering operator:

$$
C_{e 0}^{l}=\nu_{e i}(v) L \doteq \nu_{e i}(v) \frac{1}{2} \frac{\partial}{\partial \xi}\left(1-\xi^{2}\right) \frac{\partial}{\partial \xi} \quad \text { and } \quad \xi \doteq \frac{v_{\|}}{v} .
$$

The solutions of Eqs. (14a) in this approximation can be written in the following form:

$$
g_{e n}^{0}=-\sigma \frac{v}{2 \nu_{e i}(v)}\left\langle B \mathcal{C}_{e n}^{\gamma}(v, B)\right\rangle f_{e 0} \int_{\lambda}^{\lambda_{c}} \frac{d \lambda^{\prime}}{\left\langle\left(1-\lambda^{\prime} B\right)^{1 / 2}\right\rangle} H\left(\lambda_{c}-\lambda\right)
$$

where $\lambda \doteq\left(1-\xi^{2}\right) / B, \lambda_{c} \doteq 1 / B_{\max }$ and $H(x)$ is the Heaviside function. Introducing Eq. (15) in the expressions for the coefficients Eqs. (12), all the electron transport coefficients can be written as integrals in the absolute value of velocity $\mathrm{v}$ :

$$
\mathcal{L}_{m n}^{e}=\frac{4 \pi}{3} \int_{0}^{\infty} v^{4} d v\left\langle\frac{\gamma_{e m}}{v_{\|}} \mathcal{C}_{e n}^{\gamma}\right\rangle f_{e 0}+\pi \int_{0}^{\infty} \frac{v^{4} d v}{\nu_{e i}(v)}\left\langle B \mathcal{C}_{e m}^{\gamma}\right\rangle\left\langle B \mathcal{C}_{e n}^{\gamma}\right\rangle f_{e 0}, \quad n, m=1,2,3,4
$$

The integrals of $\mathrm{v}$ can easily be computed analytically, and the results have been compared with Ref. 2, Eqs. (5.121), (5.124 - 5.130), finding a complete agreement. As the Lorentz model coefficients will be used not only as a benchmark for the results of CQL3D, but also to analyze the results of the code with the full collision operator and in different axisymmetric configurations, we report all the electron transport coefficients, which can be written in the following simple form:

$$
\begin{array}{ll}
\mathcal{L}_{11}^{e}=-0.5 \mathcal{L}_{d}\left[B_{0}^{2}\left\langle B^{-2}\right\rangle\right] f_{t}^{d}, & \\
\mathcal{L}_{21}^{e}=0.75 \mathcal{L}_{d}\left[B_{0}^{2}\left\langle B^{-2}\right\rangle\right] f_{t}^{d}, & \mathcal{L}_{22}^{e}=-\frac{13}{8} \mathcal{L}_{d}\left[B_{0}^{2}\left\langle B^{-2}\right\rangle\right] f_{t}^{d}, \\
\mathcal{L}_{31}^{e}=\mathcal{L}_{34}^{e}=-\mathcal{L}_{b} f_{t}, \quad \mathcal{L}_{32}^{e}=0, & \mathcal{L}_{33}^{e}=-\frac{32}{3 \pi} \mathcal{L}_{\sigma}\left[B_{0}^{-2}\left\langle B^{2}\right\rangle\right] f_{t}, \\
\mathcal{L}_{41}^{e}=\mathcal{L}_{44}^{e}=-0.5 \mathcal{L}_{d}\left[B_{0}^{2}\left\langle B^{2}\right\rangle^{-1}\right] f_{t}, & \mathcal{L}_{42}^{e}=0.75 \mathcal{L}_{d}\left[B_{0}^{2}\left\langle B^{2}\right\rangle^{-1}\right] f_{t},
\end{array}
$$

with

$$
\mathcal{L}_{d}=\frac{n_{e} \rho_{e p}^{2}}{\tau_{e}}\left(\frac{d \psi}{d \rho}\right)^{2}, \quad \mathcal{L}_{b}=I(\psi) n_{e}, \quad \mathcal{L}_{\sigma}=\frac{n_{e} q_{e}^{2} \tau_{e}}{m_{e} T_{e}} B_{0}^{2}
$$


and where we have introduced two definitions for the trapped fraction:

$$
\begin{gathered}
f_{t}^{d}=1-\frac{3}{4}\left\langle B^{-2}\right\rangle^{-1} \mathcal{I}_{\lambda}, \quad f_{t}=1-\frac{3}{4}\left\langle B^{2}\right\rangle \mathcal{I}_{\lambda}, \\
\mathcal{I}_{\lambda}=\int_{0}^{\lambda_{c}} \frac{\lambda^{\prime} d \lambda^{\prime}}{\left\langle\left(1-\lambda^{\prime} B\right)^{1 / 2}\right\rangle} .
\end{gathered}
$$

The second one, $f_{t}$, is the usual definition for the trapped particle fraction ${ }^{3}$. Note that the integral $\mathcal{I}_{\lambda}$ can easily be evaluated using the formulas in Ref. 13. The definitions adopted for the poloidal gyroradius $\rho_{e p}$ and the electron collision time $\tau_{e}$ are the same as in Ref. 2, Eq. (5.122) and Eq. (5.4) respectively. Note that the flux surface averaged integrals $I_{11}$, $I_{13}$ and $I_{33}$ which appear in the results of Ref. 2 can be reduced to only the two trapped fractions, Eqs. (18), with the following relations:

$$
I_{11}=\frac{4}{3}\left[B_{0}^{2}\left\langle B^{-2}\right\rangle\right] f_{t}^{d}, \quad I_{13}=\frac{4}{3} f_{t}, \quad I_{33}=\frac{4}{3}\left[B_{0}^{-2}\left\langle B^{2}\right\rangle\right] f_{t} .
$$

We see therefore that all the coefficients in the Lorentz model depend essentially on $f_{t}^{d}$ and $f_{t}$. We shall show in the next Sections that in the general case this property remains true, namely that all the equilibrium effects on the neoclassical transport coefficients are funstions of only these two trapped fractions.

\section{NUMERICAL RESULTS}

\section{A. Benchmarks}

As the Lorentz model gives an analytical solution, it can be used as a first benchmark for the numerical results. In Fig. 1 we show the transport coefficients $\mathcal{L}_{1 n}^{e}$ and $\mathcal{L}_{2 n}^{e}$ relative to the sources $S_{e 1}$ and $S_{e 2}$, Eqs. (14), computed by CQL3D in the approximation of the Lorentz model: very good agreement is obtained for all $\epsilon$. The coefficients in the Figure, indicated by $L_{m n}^{e}$, are plotted normalized by the relative factors $\mathcal{L}_{d}$ or $\mathcal{L}_{b}$ given in Eqs. (17). This normalization for the electron transport cofficients is also kept in Fig. 2 and Fig. 4. The complete definition of a set of dimensionless coefficients will be given in the next section. 
When the full collision operator is used, the symmetry of the transport matrix gives a second benchmark of the numerical results: indeed, each off-diagonal coefficient can be computed in two different ways, $\mathcal{L}_{n m}$ and $\mathcal{L}_{m n}$. Note that we have already computed ${ }^{1}$ the neoclassical resisistivity and the bootstrap current coefficients $\mathcal{L}_{3 n}, n=1,2,4$. In Fig. 2(a) we plot the results for the coefficient $\mathcal{L}_{13}^{e}$, computed solving the kinetic equation with the source $S_{e 1}$, Eqs. (14a,c), $n=1$ : they are perfectly aligned with the solid line given by Eq. (14a) of Ref. 1 , which fits the code results for the bootstrap current coefficient $\mathcal{L}_{31}^{e}$, hence computed with the source $S_{e 3}$, Eqs. $(14 \mathrm{a}, \mathrm{c}), n=3$. The exact relations between the bootstrap current coefficients defined in Ref. 1 and the transport coefficients defined in this paper will be presented in the next section. In Fig. 2(b) we plot the two coefficients $\mathcal{L}_{12}^{e}$ and $\mathcal{L}_{21}^{e}$, computed considering four different equilibria, as shown in Fig. 1 of Ref. 1 . The coefficient $\mathcal{L}_{12}^{e}$, obtained solving Eq. (14a,c), $n=1$, is plotted with symbols, the coefficient $\mathcal{L}_{21}^{e}$, obtained solving Eq. $(14 \mathrm{a}, \mathrm{c}), n=2$, is plotted with solid lines: we find a very good agreement between the two coefficients, within $1 \%$ for $\epsilon \geq 0.1$. We see also that the behaviour of the transport coefficient strongly depends on the equilibrium at small aspect ratio. Previous formulas, which give the transport coefficients with an expansion in powers of $\epsilon^{1 / 2}$, are correct only for almost cylindrical equilibria and are of practical interest in general equilibria only for $\epsilon<0.1$ : this must be taken into account when comparing with our results. It indicates that for each transport coefficient an appropriate geometrical parameter, like $f_{t}$ for $\mathcal{L}_{31}^{e}$ and $\mathcal{L}_{13}^{e}$ in Fig. 2a, needs to be used instead of $\epsilon$, as it will be shown in the next subsection.

\section{B. Comparison with previous results and behaviour at small aspect ratio}

As we have said in Sec. I, the most recent investigations on perpendicular neoclassical transport were dedicated only to the ion thermal conductivity ${ }^{4-5}$. In Fig. 3 we compare our results for the coefficient $\mathcal{L}_{22}^{i}$, obtained with an almost cylindrical equilibrium, with the results of Ref. 4, 5 and 9. For easier comparison with Fig. 1 of Ref. 5, the plotted coefficient is normalized like in Ref. 5: 


$$
\bar{L}_{22}^{i}=-\mathcal{L}_{22}^{i}\left[\frac{n_{i} \rho_{i p}^{2}}{\tau_{i}}\left(\frac{d \psi}{d \rho}\right)^{2}\right]^{-1} \frac{\langle B\rangle^{2}}{B_{0}^{2}} \epsilon^{-1 / 2},
$$

and plotted versus the inverse aspect ratio $\epsilon$. We find good agreement with the results of Refs. 4 and 5 . These results enable to finally resolve the discrepancy between the formulas given in Refs. 4 and 5, obtained with approximated collision operators. It turns out that the results with the full collision operator, CQL3D, are in between the previous results. Note that the plotted formula of Ref. 9 has been modified, keeping in the expression for the transport coefficient the flux surface average of the magnetic field, which were correctly computed in the reference to obtain the limit at $\epsilon=1$, but then not taken into account in the final formulas.

When different axisymmetric equilibria are considered in the numerical calculations, the transport coefficients present particular features of the influence of geometry at small aspect ratio, as already highlighted in Fig. 2b. In Fig. 4 we also show the electron coefficient $\mathcal{L}_{22}^{e}$, computed with four different equilibria. In Fig. $4 \mathrm{a}, \mathcal{L}_{22}^{e}$ is plotted versus $\epsilon^{1 / 2}$ : we see differences up to $30 \%$ already at $\epsilon=0.15$. In Fig. $4 \mathrm{~b}$ we show the same coefficient $\mathcal{L}_{22}^{e}$ divided by the flux surface average $\left(B_{0}^{2}<B^{-2}>\right)$ and plotted versus the trapped particle fraction $f_{t}^{d}$, defined in Eq. (18), as suggested by the results of the Lorentz model: the points are well-aligned at all $f_{t}^{d}$, i.e. at all $\epsilon$. The same behaviour is obtained for all the other transport coefficients: the coefficients must be normalized by a suitable flux surface average and a correct geometrical parameter must be used to encapsulate the effects of the different equilibria. Note that the definition of a new trapped particle fraction, $f_{t}^{d}, \mathrm{Eq}$. (18), is effectively necessary, as suggested by the Lorentz model, to correctly describe the geometrical behaviour of certain coefficients, in particular all the particle and heat conductivities, for which the usual one, $f_{t}$, turns out to be inadequate. 


\section{TRANSPORT COEFFICIENTS FORMULAS}

\section{A. Analytical fits to the numerical results for the banana regime}

Considering the results of the previous section, we can introduce a set of dimensionless electron and ion transport coefficients $\mathcal{K}_{m n}^{\sigma}$ :

$$
\begin{array}{ll}
\mathcal{L}_{n m}^{e}=\mathcal{L}_{d}\left[B_{0}^{2}\left\langle B^{-2}\right\rangle\right] \mathcal{K}_{n m}^{e}\left(f_{t}^{d}\right), & n, m=1,2, \\
\mathcal{L}_{n 3}^{e}=\mathcal{L}_{b} \mathcal{K}_{n 3}^{e}\left(f_{t}\right), & n=1,2,4 \\
\mathcal{L}_{n 4}^{e}=\mathcal{L}_{d}\left[B_{0}^{2}\left\langle B^{2}\right\rangle^{-1}\right] \mathcal{K}_{n 4}^{e}\left(f_{t}\right), & n=1,2, \\
\mathcal{L}_{33}^{e}=\mathcal{L}_{\sigma}\left[B_{0}^{-2}\left\langle B^{2}\right\rangle\right] \mathcal{K}_{33}^{e}\left(f_{t}\right), & \\
\mathcal{L}_{11}^{i}=\mathcal{L}_{\sigma}^{i}\left[B_{0}^{-2}\left\langle B^{2}\right\rangle\right] \mathcal{K}_{11}^{i}\left(f_{t}\right), & \\
\mathcal{L}_{12}^{i}=\mathcal{L}_{b}^{i} \mathcal{K}_{12}^{i}\left(f_{t}\right), & \\
\mathcal{L}_{22}^{i}=\mathcal{L}_{d}^{i}\left[B_{0}^{2}\left\langle B^{-2}\right\rangle\right] \mathcal{K}_{22}^{i}\left(f_{t}^{d}\right), &
\end{array}
$$

and analogously all their symmetrics, where $\mathcal{L}_{d}, \mathcal{L}_{b}$ and $\mathcal{L}_{\sigma}$ are defined by Eqs. (17); the ion normalization factors, $\mathcal{L}_{d}^{i}, \mathcal{L}_{b}^{i}$ and $\mathcal{L}_{\sigma}^{i}$, are defined as follows:

$$
\mathcal{L}_{d}^{i}=\frac{n_{i} \rho_{i p}^{2}}{\tau_{i}}\left(\frac{d \psi}{d \rho}\right)^{2}, \quad \mathcal{L}_{b}^{i}=I(\psi) n_{i}, \quad \mathcal{L}_{\sigma}^{i}=\frac{n_{i} q_{i}^{2} \tau_{i}}{m_{i} T_{i}} B_{0}^{2}
$$

The ion-ion collision time $\tau_{i}$ is defined in Ref. 2, Eq. (4.24). The dimensionless coefficients are functions of only one suitable geometrical parameter, i.e. a trapped fraction, which completely encapsulates the effects of the various equilibria: in this way the code results for the coefficients $\mathcal{K}_{m n}^{\sigma}$ can be fitted in terms of the appropriate trapped fraction, $f_{t}$ or $f_{t}^{d}$, as they perfectly overlap, regardless the equilibrium considered in the calculation, even highly non-circular and at small aspect ratio. Note that only in this way relatively simple formulas valid in general axisymmetric equilibria and at all aspect ratios can be given. We have already computed the neoclassical conductivity and the bootstrap current coefficients, in Ref. 1, solving the same kinetic equation of Eq. (14a), $n=3$, and computing the transport coefficients with the same integrals given by Eq. (12), $n=3$, and Eq. (13), 
$n=2, m=1$, which were first obtained using an adjoint formalism ${ }^{11}$ adapted to calculate only the bootstrap current: the general kinetic results of Sec. II show however that the adjoint formulation is not necessary. The relations between the transport coefficient $\mathcal{L}_{33}^{e}$ and the neoclassical conductivity $\sigma_{n e o}$, Eq. (13a) in Ref. 1, and between the dimensionless coefficients $\mathcal{K}_{3 m}^{e}, m=1,2,4$, and the bootstrap current coefficients $\mathcal{L}_{3 m}^{b s}$, Eqs. (14) and (15) in Ref. 1, read:

$$
\mathcal{L}_{33}^{e}=\frac{\sigma_{n e o}-\sigma_{S p t z}}{T_{e}}\left\langle B^{2}\right\rangle, \quad \quad \mathcal{K}_{3 m}^{e}=-\mathcal{L}_{3 m}^{b s}
$$

The coefficient $\mathcal{K}_{12}^{i}$ is related to the coefficient $\alpha$, Eqs. (17a,b) in Ref. 1 , by Eq. (26). We have run the code CQL3D with different equilibria and for the electron coefficients we have also varied the ion charge, to obtain the dependence on the effective charge $Z$. Our idea is that, at least for the electron transport coefficients, an effective charge approximation for multispecies cases should still be valid: collisions between electrons and main ions, or between electrons and impurity ions are almost of the same kind, involving basically the pitch-angle scattering. In any case, the comparison with the results of multispecies codes $^{14}$ should enable one to determine the correct form of $Z$, instead of the usual definition of $Z_{\text {eff }}$, to be used in our formulas, as already mentioned in Ref. 1. For the ions, the presence of one heavy impurity species leads to collisions between main ions and impurity ions which involve basically the pitch-angle scattering, and which are completely different from likeparticle collisions. In this case, as shown in Ref. 15, the thermal conductivity computed as $Z_{\text {eff }}$ times the pure ion conductivity is underestimated. Using the results of Ref. 15, which uses the large aspect ratio limit of Ref. 16, we have generalized our formula for the transport coefficient $\mathcal{L}_{22}^{i}$, to include the effect of a single heavy impurity species in the Pfirsch-Schlüter regime. We have also adapted the formula for the bootstrap current coefficient $\alpha$ in the banana regime, Eq. (17a) in Ref. 1, to include the same effect, using the large aspect ratio limit of Ref. 16, and noting that at $\epsilon=1$ not only the pure plasma coefficient, but also the impurity contribution must be equal to zero. The analytical fits to the results of CQL3D for all the transport coefficients not already computed in Ref. 1, valid for arbitrary trapped 
fraction and $Z$, and the modified formula for the bootstrap current coefficient $\alpha$, read as follows:

$$
\begin{gathered}
\mathcal{K}_{11}^{e}=-0.5 F_{11}\left(f_{t}^{d}\right), \\
\mathcal{K}_{12}^{e}=0.75 F_{12}\left(f_{t}^{d}\right), \\
\mathcal{K}_{14}^{e}=\mathcal{K}_{44}^{e}=-0.5 F_{11}\left(f_{t}\right), \\
\mathcal{K}_{22}^{e}=-\left(\frac{13}{8}+\frac{\sqrt{2}}{2 Z}\right) F_{22}\left(f_{t}^{d}\right), \\
\mathcal{K}_{24}^{e}=0.75 F_{12}\left(f_{t}\right), \\
\mathcal{K}_{22}^{i}=-F_{22}^{i}\left(f_{t}^{d}\right), \\
F_{11}(X) \doteq\left(1+\frac{0.9}{Z+0.5}\right) X-\frac{1.9}{Z+0.5} X^{2}+\frac{1.6}{Z+0.5} X^{3}-\frac{0.6}{Z+0.5} X^{4}, \\
F_{12}(X) \doteq\left(1+\frac{0.6}{Z+0.5}\right) X-\frac{0.95}{Z+0.5} X^{2}+\frac{0.3}{Z+0.5} X^{3}+\frac{0.05}{Z+0.5} X^{4}, \\
F_{22}(X) \doteq\left(1-\frac{0.11}{Z+0.5}\right) X+\frac{0.08}{Z+0.5} X^{2}+\frac{0.03}{Z+0.5} X^{3}, \\
F_{22}^{i}(X) \doteq(1-0.55)\left(1+1.54 \alpha_{I}\right) X+ \\
\left(0.75 X^{2}-0.7 X^{3}+0.5 X^{4}\right)\left(1+2.92 \alpha_{I}\right), \\
\alpha=-\mathcal{K}_{12}^{i}=-\frac{0.62+1.5 \alpha_{I}}{0.53+\alpha_{I}} \frac{1-f_{t}}{1-0.22 f_{t}-0.19 f_{t}^{2}},
\end{gathered}
$$

where $\alpha_{I}=n_{I} Z_{I}^{2} / n_{i} Z_{i}^{2}$ is the usual impurity strength parameter, and index $I$ refers to the ion impurity species. The factorizations used in Eqs. (24) and (25) are such that the Lorentz limit $(Z \rightarrow \infty)$, the low $\left(f_{t} \rightarrow 0\right)$ and the large aspect ratio $\left(f_{t} \rightarrow 1\right)$ are easily recovered. Moreover the functions $F_{i j}$ have values within $[0,1]$. Note that $\mathcal{K}_{11}^{e}$ and $\mathcal{K}_{14}^{e}$, as well as $\mathcal{K}_{12}^{e}$ and $\mathcal{K}_{24}^{e}$ have the same functional dependence on their respective trapped fraction. These relations can be considered as the extension to a general axisymmetric equilibrium at all aspect ratios of Eqs. (6.28) - (6.30) and Eq. (6.47) in Ref. 2. We have also computed the coefficient $\mathcal{L}_{11}^{i}$, which is usually not considered, following the weak-coupling approximation, which neglects the force $A_{i 1}$. For completeness, we give also the fit to the code results for the transport coefficient $\mathcal{K}_{11}^{i}$ : 


$$
\mathcal{K}_{11}^{i}\left(f_{t}\right)=\left(0.11+1.7 f_{t}-1.25 f_{t}^{2}+0.44 f_{t}^{3}\right)^{-1}-1
$$

Note that Eqs. (6) allow to reduce the number of independent thermodynamic forces form 6 to 4 , hence with only 4 conjugated thermodynamic fluxes. Taking the first 3 electron forces and the second ion force, whose conjugated fluxes have more direct physical meaning and more direct application in the fluid transport equations, the relations which connect fluxes with forces read as follows:

$$
\begin{aligned}
& B_{e n}=\sum_{m=1}^{3}\left\{\mathcal{L}_{n m}^{e}-\frac{1}{\mathcal{F}} \frac{T_{i}}{Z_{i}^{2} T_{e}} \frac{\mathcal{L}_{11}^{i} \mathcal{L}_{n 4}^{e}}{\left[I(\psi) n_{i}\right]^{2}}\right\} A_{e m}-\frac{1}{\mathcal{F}} \frac{T_{i}}{Z_{i} T_{e}} \mathcal{L}_{n 4}^{e} \frac{\mathcal{L}_{12}^{i}}{I(\psi) n_{i}} A_{i 2}, \quad n=1,2,3 \\
& B_{i 2}=\frac{1}{\mathcal{F}} \frac{\mathcal{L}_{21}^{i}}{I(\psi) n_{i}} \sum_{m=1}^{3} \mathcal{L}_{4 m}^{e} A_{e m}+\left\{\mathcal{L}_{22}^{i}-\frac{1}{\mathcal{F}} \frac{T_{i}}{Z_{i}^{2} T_{e}} \frac{\mathcal{L}_{12}^{i} \mathcal{L}_{21}^{i}}{\left[I(\psi) n_{i}\right]^{2}} \mathcal{L}_{44}^{e}\right\} A_{i 2}
\end{aligned}
$$

where

$$
\mathcal{F} \doteq 1+\frac{T_{i}}{Z_{i}^{2} T_{e}} \frac{\mathcal{L}_{11}^{i} \mathcal{L}_{44}^{e}}{\left[I(\psi) r_{i}\right]^{2}}
$$

and $Z_{i}$ is the main ion charge number. The condition for the validity of the weak-coupling approximation is given by Eq. (5.86) in Ref. 2 , and is simply $\mathcal{F}-1 \ll 1$. Introducing the dimensionless coefficients $\mathcal{K}_{m n}^{\sigma}$, this relation reads, consistently with the estimate given in Ref. 2, Table IV:

$$
\frac{2 \sqrt{2}}{Z_{i}}\left(\frac{m_{e}}{m_{i}}\right)^{\frac{1}{2}}\left(\frac{T_{i}}{T_{e}}\right)^{\frac{3}{2}} \mathcal{K}_{14}^{e} \mathcal{K}_{11}^{i} \ll 1 .
$$

The absolute value of the term $\mathcal{K}_{14}^{e} \mathcal{K}_{11}^{i}$ turns out to be smaller then 0.25 , which confirms the validity of the weak coupling approximation in the banana regime. In this way Eqs. (27) are reduced to:

$$
\begin{aligned}
B_{e n} & =\sum_{m=1}^{3} \mathcal{L}_{n m}^{e} A_{e m}-\frac{T_{i}}{Z_{i} T_{e}} \mathcal{L}_{n 4}^{e} \frac{\mathcal{L}_{12}^{i}}{I(\psi) n_{i}} A_{i 2}, \quad n=1,2,3 \\
B_{i 2} & =\frac{\mathcal{L}_{21}^{i}}{I(\psi) n_{i}} \sum_{m=1}^{3} \mathcal{L}_{4 m}^{e} A_{e m}+\left\{\mathcal{L}_{22}^{i}-\frac{T_{i}}{Z_{i}^{2} T_{e}} \frac{\mathcal{L}_{12}^{i}\left[\left(\mathcal{L}_{21}^{i}\right.\right.}{\left[I\left(\psi n_{i}\right]^{2}\right.} \mathcal{L}_{44}^{e}\right\} A_{i 2}
\end{aligned}
$$

In Figure 5 we compare the code results for the dimensionless transport coefficients $\mathcal{K}_{n m}^{\sigma}$ (symbols) with the algebraic formulas, Eqs. (24) and (25), which fit the data, (solid lines). 


\section{B. Tentative formulas for all collisionality regimes}

In order to compute the neoclassical transport coefficients at arbitrary collisionality regime, the non-bounce-averaged kinetic equations, Eqs. (10) and (11), must be solved. This, as already mentioned, has been done in Ref. 1, to compute the neoclassical resitivity and all the bootstrap current coefficients, using the code CQLP, which includes the advection parallel to the magnetic field, without any assumption on the ratio between the collision frequency and the bounce frequency. In Fig. 6 we compare the formulas of Ref. 1 (solid lines) with those, valid for all collisionality regimes at large aspect ratio, of Ref. 2, Sec. VI F, (dashed lines), in which we have replaced the banana limit with the correct results of the code CQL3D, valid at all aspect ratios: in this way we strictly compare only the dependence on collisionality, as an important contribution to the difference at arbitrary collisionality regimes comes directly from the error at the banana limit. The neoclassical resistivity is shown in Fig. 6(a) and the bootstrap current coefficient $\mathcal{L}_{31}^{e}$ in Fig. 6(b), for various values of the trapped fraction. At large aspect ratio, i.e. at small values of the trapped fraction, good agreement is found, as it must be expected; instead at larger values of the trapped fraction, where the results of Ref. 2 can no more be supposed valid, large errors arise, already at low collisionality. Indeed, the main approximation in the banana-plateau regime, computed in Ref. 17, comes from the model collision operator, obtained keeping only the first term in an expansion in powers of $\epsilon^{1 / 2}$, and leading to the pitch-angle collision operator also for the like-particle collision operator, hence neglecting the energy scattering collisions: at small aspect ratio this approximation is no more valid and underestimates the neoclassical contribution of the transport coefficients ${ }^{6}$. However both the neoclassical resistivity and the bootstrap current coefficient $\mathcal{L}_{31}^{e}$, and also the coefficient $\mathcal{L}_{32}^{e}$ not shown here, have approximatively the same behaviour at small aspect ratio: the Ref. 2 formulas go down to zero at smaller values of $\nu_{e *}$ with respect to the rigorous results of Ref. 1 . Hence the dependence on collisionality of all these coefficients at small aspect ratio can be approximatively described by the formulas of Ref. 2 , if the collisional parameter $\nu_{e *}$ is adequately rescaled in terms of 
the trapped fraction. We see that the simple transformation

$$
\nu_{\sigma f *}=\frac{\nu_{\sigma *}}{1+7 f_{t}^{2}}
$$

allows an agreement within $20 \%$ for all the bootstrap current coefficients and the neoclassical resistivity (dashed-dotted lines), when compared with Ref. 1. Hence, following the idea of Ref. 4 , in which a formula valid for all collisionality regimes for the ion heat conductivity is obtained connecting a new banana limit, valid also at small aspect ratio, with the collisional dependence of Ref. 2, we suggest to use formulas of Ref. 2, Sec. VI F, replacing the results of this paper in the limit at $\nu_{\sigma *}=0$. The transport coefficients $\mathcal{K}_{m n}^{e}, m, n=1,2$ and $\mathcal{K}_{22}^{i}$ can be computed at arbitrary collisionality regimes as follows:

$$
\begin{gathered}
\mathcal{K}_{11}^{e}=\mathcal{H}_{11}^{e}, \quad \mathcal{K}_{12}^{e}=\mathcal{H}_{12}^{e}-\frac{5}{2} \mathcal{H}_{11}^{e}, \quad \mathcal{K}_{22}^{e}=\mathcal{H}_{22}^{e}-5 \mathcal{H}_{12}^{e}+\frac{25}{4} \mathcal{H}_{11}^{e}, \quad \mathcal{K}_{22}^{i}=\mathcal{H}_{22}^{i} \\
\mathcal{H}_{m n}^{\sigma}=\frac{\mathcal{H}_{m n}^{\sigma(0)}}{1+a_{m n} \nu_{\sigma f *}^{1 / 2}+b_{m n} \nu_{\sigma f *}}-\frac{K_{m n}^{(0)}}{2} \frac{\epsilon^{3 / 2}\left(c_{m n}^{2} / b_{m n}\right) \nu_{\sigma f *} \epsilon^{3 / 2}}{1+c_{m n} \nu_{\sigma f *} \epsilon^{3 / 2}} F_{P S}
\end{gathered}
$$

and the coefficients $\mathcal{K}_{4 n}^{e}$ :

$$
\begin{gathered}
\mathcal{K}_{41}^{e}=\mathcal{H}_{41}^{e}, \quad \mathcal{K}_{42}^{e}=\mathcal{H}_{42}^{e}-\frac{5}{2} \mathcal{H}_{41}^{e}, \\
\mathcal{H}_{4 n}^{\sigma}=\left[\frac{\mathcal{H}_{4 n}^{\sigma(0)}}{1+a_{1 n} \nu_{\sigma f *}^{1 / 2}+b_{1 n} \nu_{\sigma f *}}-\frac{K_{1 n}^{(0)}}{2} \frac{\epsilon^{3 / 2}\left(c_{1 n}^{2} / b_{1 n}\right) \nu_{\sigma f *} \epsilon^{3 / 2}}{1+c_{1 n} \nu_{\sigma f *} \epsilon^{3 / 2}} F_{P S}^{(4)}\right] \frac{1}{1+\nu_{\sigma f *}^{2} \epsilon^{3}},
\end{gathered}
$$

with

$$
F_{P S}=1-\frac{1}{\left\langle B^{2}\right\rangle\left\langle B^{-2}\right\rangle}, \quad F_{P S}^{(4)}=\left\langle B^{-2}\right\rangle\left\langle B^{2}\right\rangle-1
$$

and where the coefficients $a_{m n}, b_{m n}, c_{m n}$ and $K_{m n}^{(0)}$ are given in Ref. 2, Table III for the electron coefficients and below Eq. (6.133) for the ion coefficient. The banana limit values $\mathcal{H}_{m n}^{e(0)}$ can be computed inverting Eqs. (31a,c) and using Eqs. (24) and (25). For the ion heat conducitvity, $\mathcal{K}_{22}^{i}$, and in the presence of one impurity species, the ion collisionality in Eq. (30) should be modified to $\nu_{i *}\left(1+1.54 \alpha_{I}\right)$, according to Ref. 15 ; also a factor needs to be added to the second term of Eq. (31b), which can readily be found in Ref. 15 . Note that we have left unchanged the dependence on $\epsilon$ for the plateau-collisional regime, computed in Ref. 18, as we have not enough information at the moment to modify it. However we have 
taken into account, for arbitrary aspect ratio, like in Ref. 4, the complete expression of the Pfirsch-Schlüter geometrical factor, by means of the terms $F_{P S}$ and $F_{P S}^{(4)}$, Eqs. (31e).

\section{CONCLUSION}

We have presented an approach for the neoclassical transport theory which allows to obtain simple equations suited for implementation in numerical codes in order to compute all the neoclassical transport coefficients. The code CQL3D, solving the bounce-averaged linearized drift-kinetic Fokker-Planck equation with the full collision operator, has been modified to calculate all these coefficients at all aspect ratios of various axisymmetric equilibria in the banana regime. Interesting features of the behaviour of the coefficients at small aspect ratio have been investigated and a set of formulas which fit the numerical results is proposed, to evaluate any transport coefficient for every axisymmetric equilibrium and at all aspect ratios. The thermodynamic fluxes:

$$
B_{e 1}=\Gamma_{e} \frac{d \psi}{d \rho}, \quad B_{e 2}=\frac{Q_{e}}{T_{e}} \frac{d \psi}{d \rho}, \quad B_{e 3}=\frac{\left\langle j_{\|} B\right\rangle}{T_{e}}-\frac{\left\langle j_{\| S} B\right\rangle}{T_{e}}, \quad B_{i 2}=\frac{Q_{i}}{T_{i}} \frac{d \psi}{d \rho}
$$

where $\Gamma_{e}$ is the perpendicular electron particle flux, $Q_{e}$ is the electron perpendicular heat flux, $j_{\|}$and $j_{\| S}$ are the parallel electric current and the Spitzer current, and $Q_{i}$ is the ion perpendicular heat flux, are given by Eqs. (29), in the weak coupling approximation, whose validity is confirmed by Eq. (28). Eqs. (29) can be reordered, and the thermodynamic fluxes can be expressed directly in terms of the electron and ion temperature and density perpendicular gradients and the parallel electric field:

$$
\begin{aligned}
B_{e n}=\mathcal{L}_{n 1}^{e} \frac{\partial \ln n_{e}}{\partial \psi}+\left(\mathcal{L}_{n 1}^{e}+\mathcal{L}_{n 2}^{e}\right) \frac{\partial \ln T_{e}}{\partial \psi}+\frac{1-R_{p e}}{R_{p e}} \mathcal{L}_{n 1}^{e} \frac{\partial \ln n_{i}}{\partial \psi}+ \\
\frac{1-R_{p e}}{R_{p e}}\left(\mathcal{L}_{n 1}^{e}+\alpha \mathcal{L}_{n 4}^{e}\right) \frac{\partial \ln T_{i}}{\partial \psi}+\mathcal{L}_{n 3}^{e} \frac{\left\langle E_{\|} B\right\rangle}{\left\langle B^{2}\right\rangle}, \quad n=1,2,3, \\
B_{i 2}=\frac{Q_{i}}{T_{i}} \frac{d \psi}{d \rho}=\alpha\left[\mathcal{L}_{41}^{e} \frac{\partial \ln n_{e}}{\partial \psi}+\left(\mathcal{L}_{41}^{e}+\mathcal{L}_{42}^{e}\right) \frac{\partial \ln T_{e}}{\partial \psi}+\mathcal{L}_{41}^{e} \frac{1-R_{p e}}{R_{p e}} \frac{\partial \ln n_{i}}{\partial \psi}+\mathcal{L}_{43}^{e} \frac{\left\langle E_{\|} B\right\rangle}{\left\langle B^{2}\right\rangle}\right]+ \\
\left(\mathcal{L}_{22}^{i}+\frac{1-R_{p e}}{R_{p e}} \frac{\alpha^{2}}{Z_{i}} \mathcal{L}_{44}^{e}\right) \frac{\partial \ln T_{i}}{\partial \psi}
\end{aligned}
$$


where $R_{p e} \doteq p_{e} / p$ and $Z_{i}$ is the main ion charge number. The transport coefficients $\mathcal{L}_{m n}^{\sigma}$ in the banana regime, for general axisymmetric equilibria, are given by Eqs. (21 - 25) of Sec. IV in terms of the trapped fractions $f_{t}$ or $f_{t}^{d}$, Eq. (18), and the effective charge number Z. Extension of this work is to compute the transport coefficients at all collisionality regimes: note that this has already been done for the neoclassical conductivity and the bootstrap current coefficients in Ref. 1. These results, compared with the ones of Ref. 2 and Ref. 4, have motivated us to propose tentative formulas for all the other transport coefficients, Eqs. (31), valid for arbitrary collisionality regime.

\section{ACKNOWLEDGMENTS}

We are grateful to Y.R. Lin-Liu and J. Vaclavik for interesting discussions. One of the authors, C.A., would also like to thank P. Helander for useful discussions. This work was partly supported by the Swiss National Science Foundation.

\section{APPENDIX A:}

\section{ONSAGER SYMMETRY OF THE TRANSPORT COEFFICIENTS}

The expressions for the transport coefficients given by Eqs. (12) for the electrons and by Eqs. (13) for the ions satisfy the Onsager relations of symmetry, as expected ${ }^{2}$. We begin with the electron case. In Eq. (12), the first term, $\mathcal{L}_{m n}^{e}{ }^{(1)} \doteq\left\langle\int d \mathbf{v} \gamma_{e m} C_{e 0}^{l}\left(\gamma_{e n} f_{e 0}\right)\right\rangle$, is symmetric directly from the self-adjointness of the collision operator. Hence:

$$
\mathcal{L}_{m n}^{e}{ }^{(1)} \doteq\left\langle\int d \mathbf{v} \gamma_{e m} C_{e 0}^{l}\left(\gamma_{e n} f_{e 0}\right)\right\rangle=\left\langle\int d \mathbf{v} \gamma_{e n} C_{e 0}^{l}\left(\gamma_{e m} f_{e 0}\right)\right\rangle \doteq \mathcal{L}_{n m}^{e}{ }^{(1)}
$$

For the second term, $\mathcal{L}_{m n}^{e}{ }^{(2)} \doteq\left\langle\int d \mathrm{v} g_{e m} / f_{e 0} C_{e 0}^{l}\left(\gamma_{e n} f_{e 0}\right)\right\rangle$, we shall rewrite it in a symmetric form. Introducing the following notation ${ }^{2}$, for a generic function $f(\mathbf{v})$, $f^{+} \doteq \frac{1}{2}[f(\sigma=+1)+f(\sigma=-1)]$ is its even part in $\sigma=v_{\|} /\left|v_{\|}\right|$and $f^{-} \doteq$ $\frac{1}{2}[f(\sigma=+1)-f(\sigma=-1)]$ is the odd part, so that: 


$$
\begin{aligned}
& \left|v_{\|}\right| \hat{\mathbf{b}} \cdot \nabla g_{e n}^{+}-C_{e 0}^{l}\left(g_{e n}^{-}\right)=-C_{e 0}^{l}\left(\gamma_{e n} f_{e 0}\right), \\
& \left|v_{\|}\right| \hat{\mathbf{b}} \cdot \nabla g_{e n}^{-}-C_{e 0}^{l}\left(g_{e n}^{+}\right)=0
\end{aligned}
$$

(as $C_{e 0}^{l}\left(\gamma_{e n} f_{e 0}\right)^{-}=C_{e 0}^{l}\left(\gamma_{e n} f_{e 0}\right)$ and $\left.C_{e 0}^{l}\left(\gamma_{e n} f_{e 0}\right)^{+}=0, n=1,2,3,4\right)$,

we can perform the following derivation:

$$
\begin{aligned}
& \mathcal{L}_{m n}^{e}{ }^{(2)} \doteq\left\langle\int d \mathbf{v} \frac{g_{e m}}{f_{e 0}} C_{e 0}^{l}\left(\gamma_{e n} f_{e 0}\right)\right\rangle=\left\langle\int d \mathbf{v} \frac{g_{e m}^{-}}{f_{e 0}} C_{e 0}^{l}\left(\gamma_{e n} f_{e 0}\right)^{-}\right\rangle= \\
& -\left\langle\int d \mathbf{v} \frac{g_{e m}^{-}}{f_{e 0}}\left[v_{\|} \hat{\mathbf{b}} \cdot \nabla g_{e n}^{+}-C_{e 0}^{l}\left(g_{e n}^{-}\right)\right]\right\rangle= \\
& -\left\langle\int d \mathbf{v} \frac{1}{f_{e 0}}\left[-g_{e m}^{+}\left|v_{\|}\right| \hat{\mathbf{b}} \cdot \nabla g_{e n}^{-}-g_{e m}^{-} C_{e 0}^{l}\left(g_{e n}^{-}\right)\right]\right\rangle= \\
& -\left\langle\int d \mathbf{v} \frac{1}{f_{e 0}}\left[-g_{e m}^{+} C_{e 0}^{l}\left(g_{e n}^{+}\right)-g_{e m}^{-} C_{e 0}^{l}\left(g_{e n}^{-}\right)\right]\right\rangle= \\
& \left\langle\int d \mathbf{v} \frac{1}{f_{e 0}} g_{e m} C_{e 0}^{l}\left(g_{e n}\right)\right\rangle,
\end{aligned}
$$

which is a symmetric expression, using the self-adjointness of the collision operator.

(Note that we have used in this derivation the fact that the operator $-v_{\|} \hat{\mathbf{b}} \cdot \nabla$ is the adjoint of the operator $\left.v_{\|} \hat{\mathbf{b}} \cdot \nabla\right)$.

Hence we can conclude that:

$$
\mathcal{L}_{m n}^{e}{ }^{(2)}=\left\langle\int d \mathbf{v} \frac{1}{f_{e 0}} g_{e m} C_{e 0}^{l}\left(g_{e n}\right)\right\rangle=\left\langle\int d \mathbf{v} \frac{1}{f_{e 0}} g_{e n} C_{e 0}^{l}\left(g_{e m}\right)\right\rangle=\mathcal{L}_{n m}^{e}{ }^{(2)}
$$

which shows the symmetry of the coefficients $\mathcal{L}_{m n}^{e}$.

A somewhat analogous calculation can be performed for the ion coefficients $\mathcal{L}_{12}^{i}$ and $\mathcal{L}_{21}^{i}$, which shows that the two given expressions, Eqs. (13), satisfy the following relation:

$$
\mathcal{L}_{12}^{i}=-\mathcal{L}_{21}^{i}
$$

consistently with the result in Ref. 2, Eq. (5.99). 


\section{REFERENCES}

${ }^{1}$ O. Sauter, C. Angioni and Y. R. Lin-Liu, Phys. Plasmas 62834 (1999).

${ }^{2}$ F. L. Hinton and R. D. Hazeltine, Rev. Mod. Phys. 48, 239 (1976).

${ }^{3}$ S. P. Hirshman and D. J. Sigmar, Nucl. Fusion 21, 1079 (1981).

${ }^{4}$ C. S. Chang and F. L. Hinton, Phys. Fluids 25, 1493 (1982).

${ }^{5}$ M. Taguchi, Phys. Contr. Fus. 30, 1897 (1988).

${ }^{6}$ C. Bolton and A. A. Ware, Phys. Fluids 26, 459 (1983).

${ }^{7}$ S. P. Hirshman and D. J. Sigmar, Phys. Fluids 19, 1532 (1976).

${ }^{8}$ R. W. Harvey and M. G. McCoy, in Proc. IAEA TCM on Advances in Simulation and Modeling of Thermonuclear Plasmas, Montreal, 1992, p. 489-526, IAEA, Vienna (1993).

${ }^{9}$ R. D. Hazeltine, F. L. Hinton and M. N. Rosenbluth, Phys. Fluids 16, 1645 (1973).

${ }^{10}$ M. N. Rosenbluth, R. D. Hazeltine and F. L. Hinton, Phys. Fluids 15, 116 (1972).

${ }^{11}$ Y. R. Lin-Liu, F. L. Hinton, C. F.F. Karney and S. P. Hirshman, International Sherwood Theory Conference, Dallas, Texas (1994) paper 3C37.

${ }^{12}$ P. H. Rutherford, Phys. Fluids 13, 482 (1970).

${ }^{13}$ Y. R. Lin-Liu and R. L. Miller, Phys. Plasmas 2, 1666 (1995).

${ }^{14}$ W. A. Houlberg, K. C. Shaing, S. P. Hirshman, and M. C. Zarnstorff, Phys. Plasmas 4, 3230 (1997).

${ }^{15}$ C. S. Chang and F. L. Hinton, Phys. Fluids 29, 1493 (1986).

${ }^{16}$ S. P. Hirshman, Phys. Fluids 19, 155 (1976).

${ }^{17}$ F. L. Hinton and M. N. Rosenbluth, Phys. Fluids 16, 836 (1973).

${ }^{18}$ J. M. Rawls, M. S. Chu and F. L. Hinton, Phys. Fluids 18, 1160 (1975). 


\section{FIGURES}

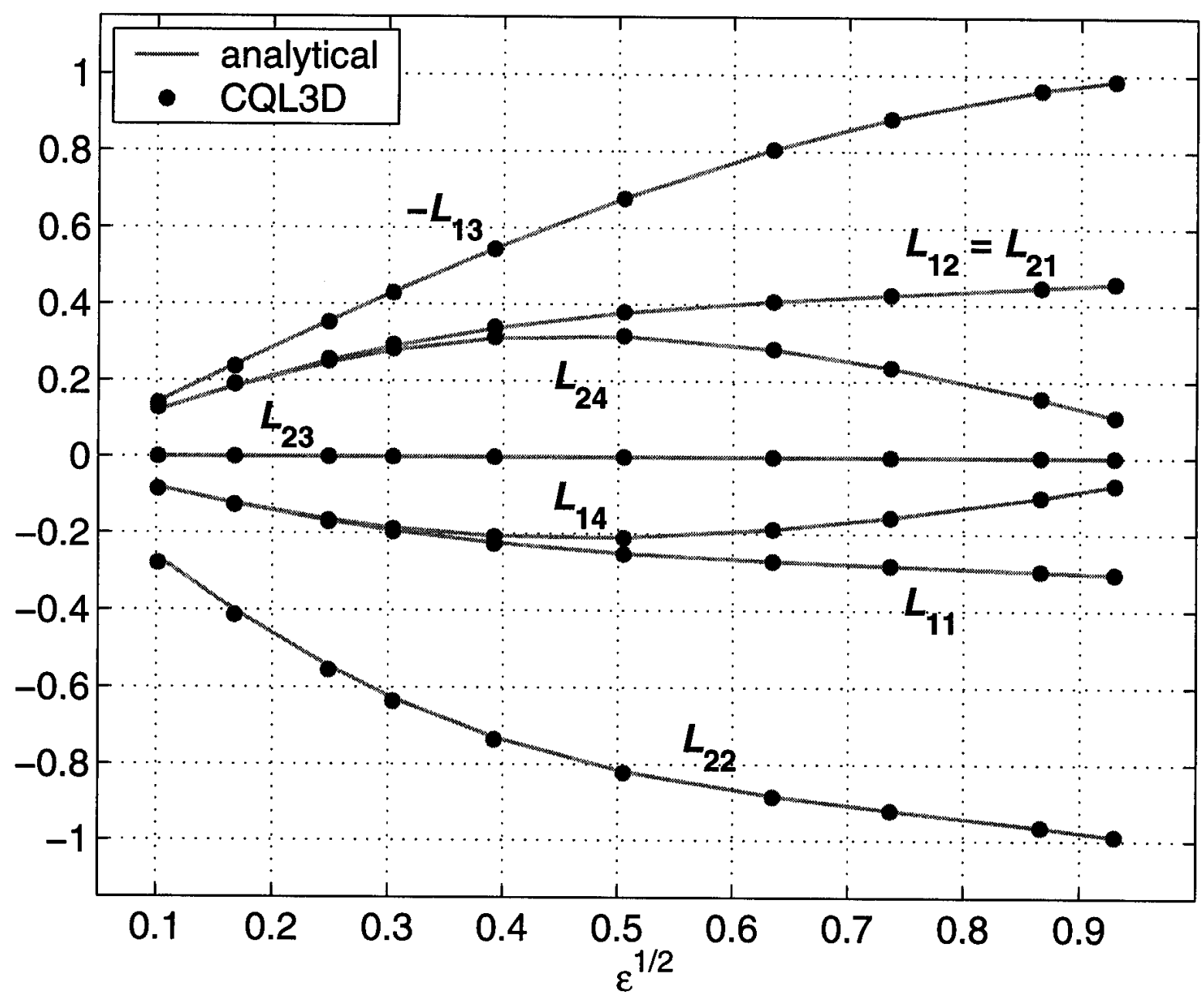

FIG. 1. Transport coefficients $\mathcal{L}_{1 n}^{e}$ and $\mathcal{L}_{2 n}^{e}$ for an almost cylindrical equilibrium, computed by CQL3D in the approximation of the Lorentz model (circles), and compared with the analytical results, Eq. (16). 

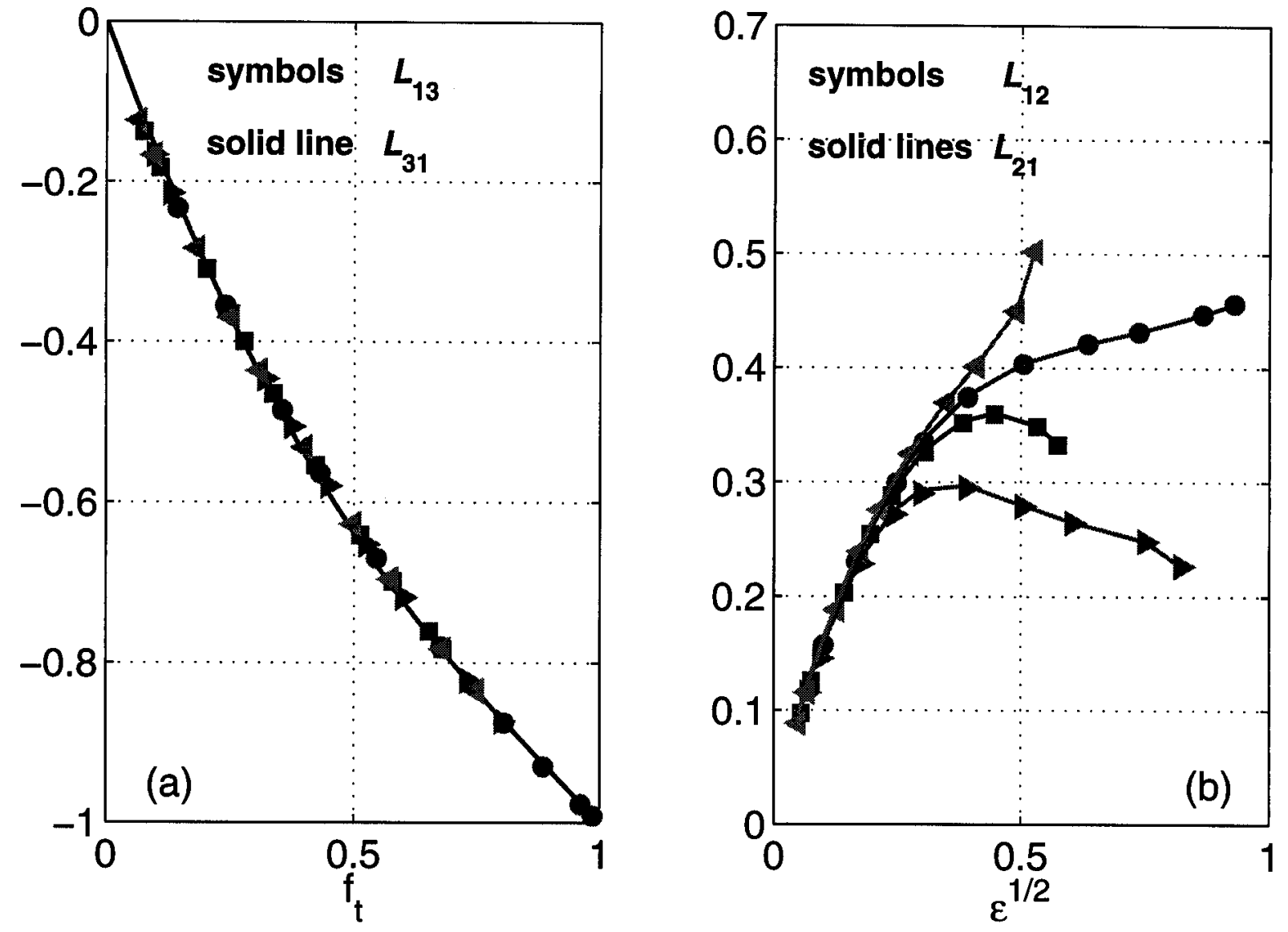

FIG. 2. Onsager symmetry is correctly respected by the numerical results. (a) The transport coefficient $\mathcal{L}_{13}^{e}$ (symbols), plotted vs $f_{t}$, is well aligned with the formula (14a) of Ref. 1 for the bootstrap current coefficient $\mathcal{L}_{31}^{e}$ (solid line). The different symbols refer to different equilibria in all the Figures. Note that the results given by the different equilibria are perfectly overlapped, as they are plotted vs $f_{t}{ }^{1}$. (b) Transport coefficient $\mathcal{L}_{12}^{e}$ (symbols) and $\mathcal{L}_{21}^{e}$ (solid lines) computed with four different equilibria, plotted vs $\epsilon^{1 / 2}$. Note that when the complete coefficients are plotted vs the inverse aspect ratio, a strong dependence on equilibria appears at small aspect ratio. 


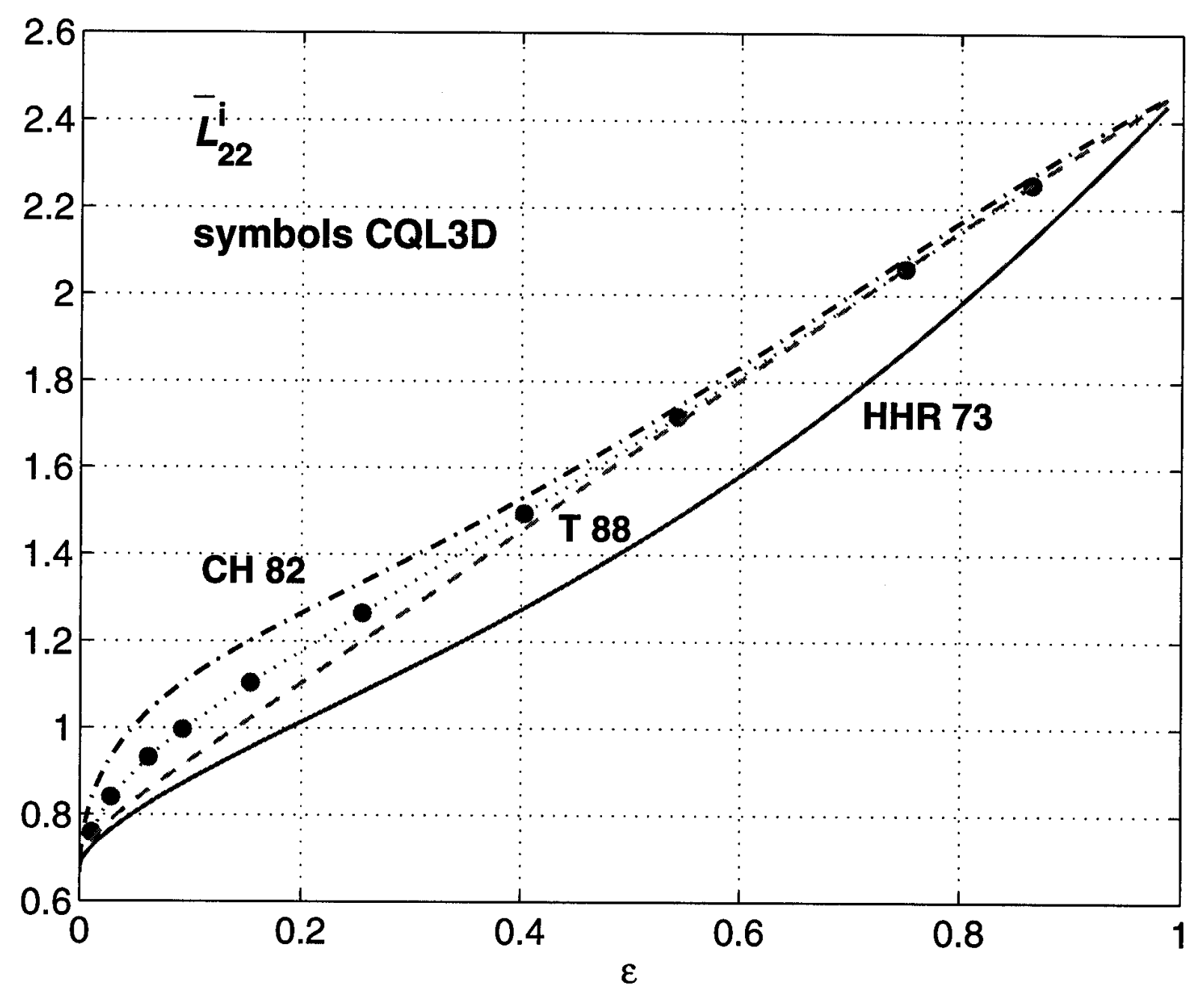

FIG. 3. The ion heat conductivity, transport coefficient $\mathcal{L}_{22}^{i}$, computed by CQL3D with an almost cylindrical equilibrium, solid circles, normalized like in Eq. (20) and plotted vs $\epsilon$, compared with the formulas of Ref. 9 (modified), HHR 73, solid line, Ref. 4, CH 82, dashed-dotted line, Ref. 5 , T 88 , dashed line. 

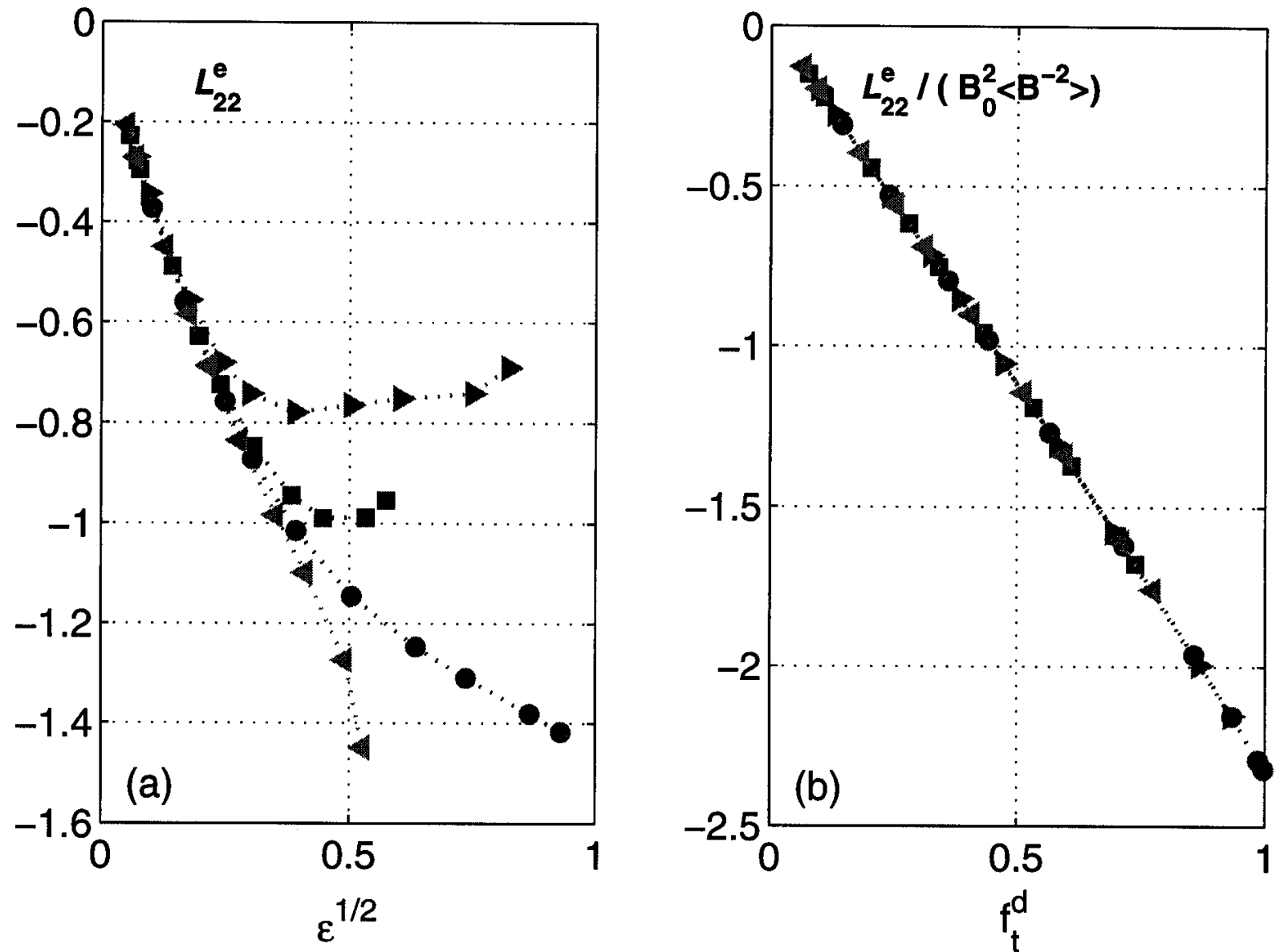

FIG. 4. The transport coefficient $\mathcal{L}_{22}^{e}$, main contribution to the electron heat conductivity, computed by CQL3D with four different equilibria: (a) the complete coefficient is plotted vs $\epsilon^{1 / 2}$, a strong dependence on the different equilibria appears at small aspect ratio; (b) the coefficient is divided by an appropriate flux surface average $B_{0}^{2}\left\langle B^{-2}\right\rangle$, and plotted vs the correct geometrical parameter, $f_{t}^{d}$, which allows to perfectly align all the points of the different equilibria. 

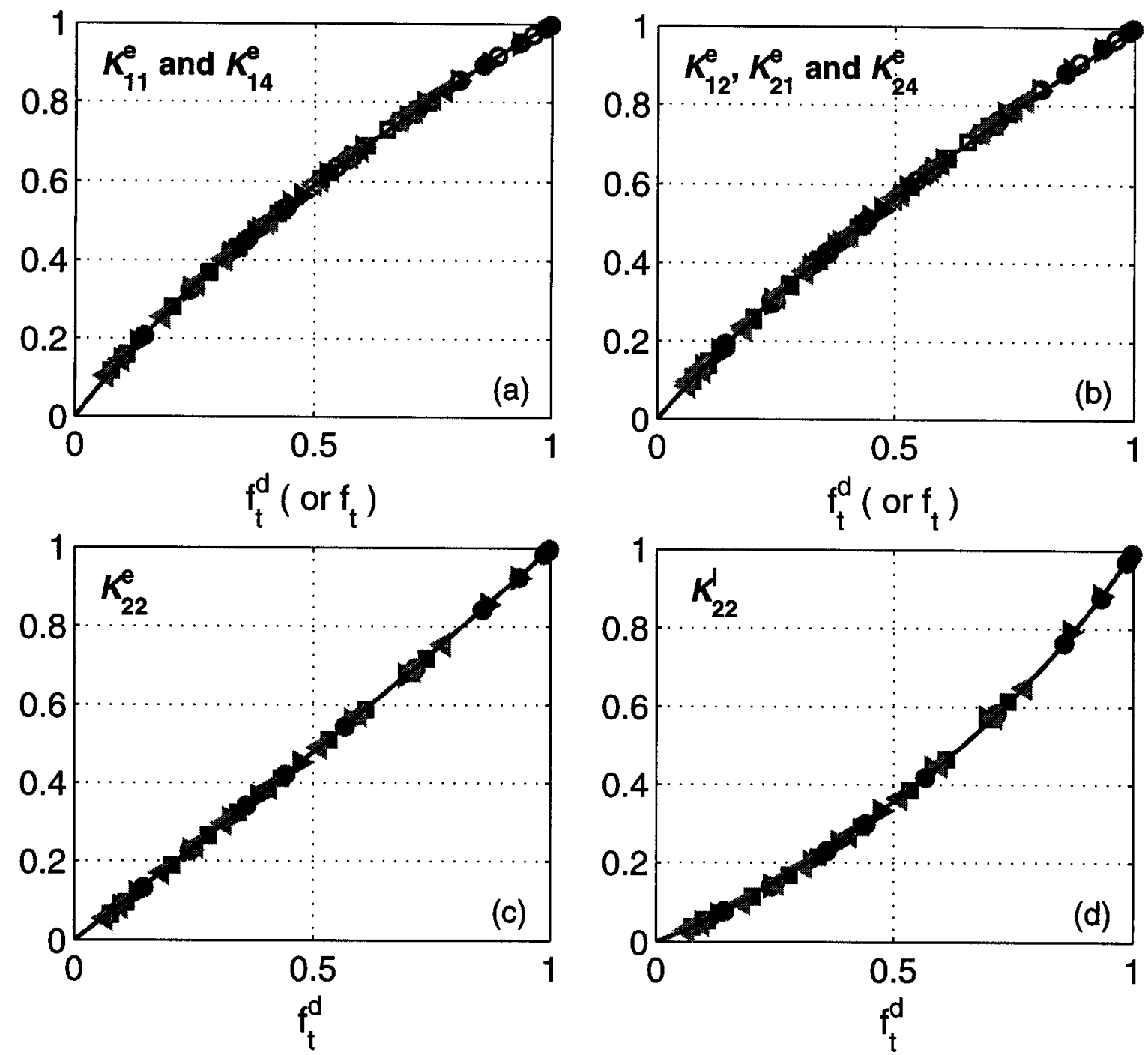

FIG. 5. Computed values of the dimensionless transport coefficients $\mathcal{K}_{m n}^{\sigma}$ (symbols), compared with the fitting formulas, Eqs. (24) and (25), (solid lines). (a) Coefficients $\mathcal{K}_{11}^{e}$ plotted vs $f_{t}^{d}$, (solid symbols), and $\mathcal{K}_{14}^{e}$ plotted vs $f_{t}$, (open symbols). (b) Coefficients $\mathcal{K}_{12}^{e}$ and $\mathcal{K}_{21}^{e}$, plotted vs $f_{t}^{d}$, (solid symbols), and $\mathcal{K}_{24}^{e}$ plotted vs $f_{t}$, (open symbols). (c) Coefficient $\mathcal{K}_{22}^{e}$ plotted vs $f_{t}^{d}$, (solid symbols). (d) Coefficient $\mathcal{K}_{22}^{i}$ plotted vs $f_{t}^{d}$, (solid symbols). 

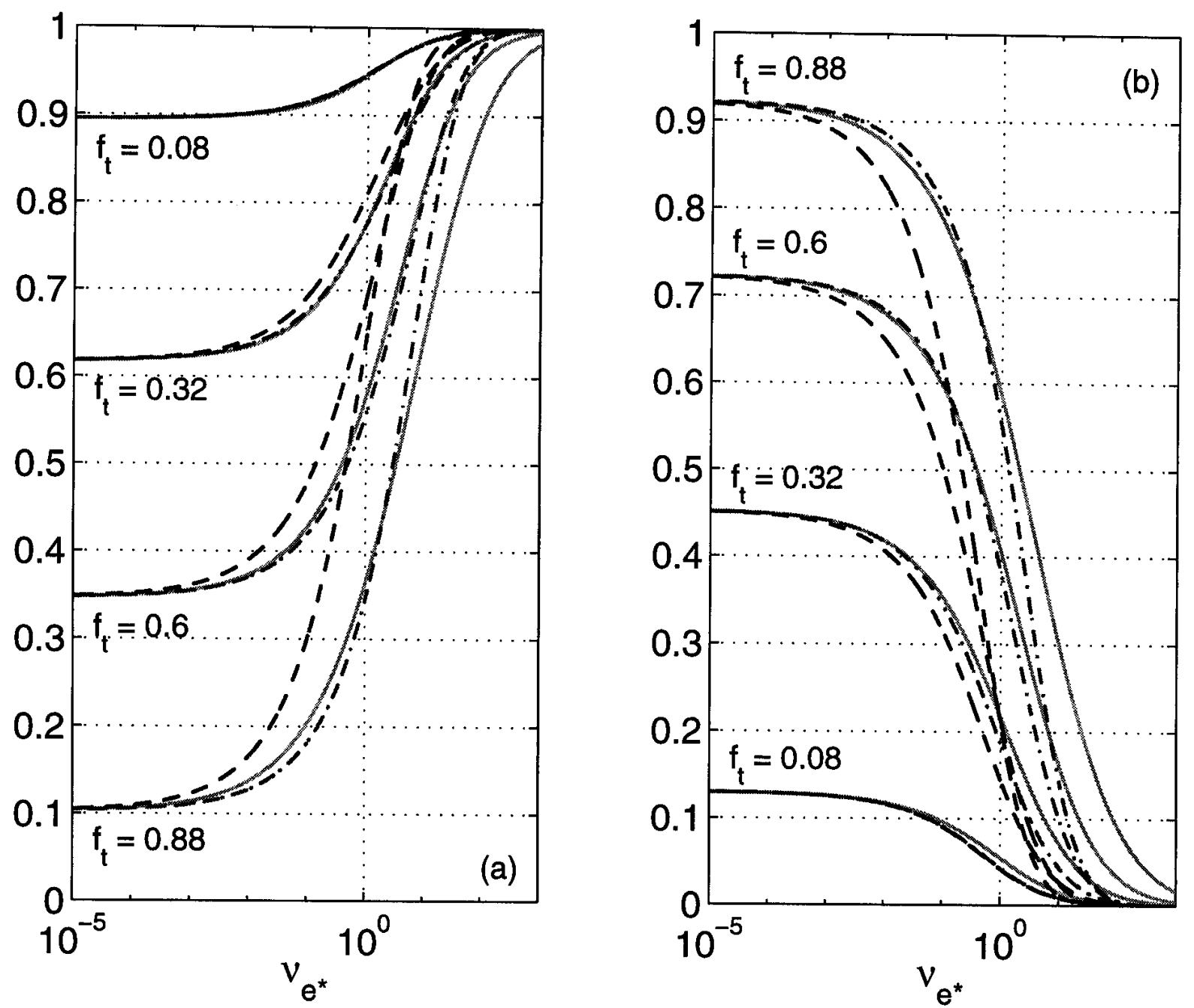

FIG. 6. Dependence on collisionality for the transport coefficients $\sigma_{n e o}$, (a), and $\mathcal{L}_{31}^{e}$, (b), for different values of the trapped fraction $f_{t}$, as given by Ref. 1, (solid lines), by Ref. 2, with the value at $\nu_{e *}=0$, banana limit, corrected with the results of Ref. 1, (dashed lines), and still by Ref. 2, with also the collisional parameter rescaled by Eq. (30), (dashed-dotted lines). 